\title{
UTILIZAÇÃO DO TURBO COMPRESSOR EM MOTORES DE COMBUSTÃO INTERNA E SEUS BENEFÍCIOS
}

Layla Fernandes Moreira, Robson dos Santos, Vinícius Bicalho da Silva e Vinícius

Ramos dos Santos

Centro Universitário de Barra Mansa

E-mails: layla.moreira@icloud.com, robsonengenharia2019@gmail.com, vbsfar@hotmail.com, vinicius-rsantos@hotmail.com

\section{RESUMO}

A sociedade moderna tem se tornado, intrinsecamente, dependente de veículos automotores, que em sua maior parte utilizam motores de combustão interna para transformar energia proveniente de reações químicas em energia mecânica, gerando movimento. Como este tipo de conversão gera poluentes, os problemas causados pelo excesso de gases liberados na atmosfera têm se tornado cada vez mais graves, resultando em diversos danos ambientais e de saúde pública em escala global. Tendo em vista tal problema, este trabalho visa realizar um estudo abordando o uso de turbo compressores em motores de pequeno porte presentes em veículos de passeio, aumentando sua potência, com baixo consumo de combustíveis e emissão de gases poluentes, o que gera vantagens para o meio ambiente e satisfação ao consumidor final. Este estudo propõe também uma contribuição para o entendimento do funcionamento técnico do sistema de indução forçada (FIS), no qual o turbo compressor é baseado, analisando as vantagens de sua aplicação e até mesmo possíveis problemas e desafios para a utilização do mesmo. 


\section{INTRODUÇÃO}

Veículos automotores utilizam, em sua maioria, motores de combustão interna conhecidos como motores de ciclo Otto [1], conhecidos como motores de quatro tempos. Esses motores funcionam como conversores de uma fonte de energia, um combustível, para energia mecânica, gerando movimento. Em 2011 as pesquisas realizadas pelo Departamento Nacional de Trânsito [2] indicavam que o Brasil possuía uma frota aproximada de 71 milhões de veículos, tendo aumentado em cerca de $121 \%$ em relação ao ano de 2001.

O uso destes motores causa uma liberação de poluentes na atmosfera, devido a queima de combustíveis, que são extremamente prejudiciais ao ambiente e a saúde da população. Tendo em vista tal problema, autoridades de todo o mundo estão criando regulamentações e exigências para que sejam realizadas diminuições nas emissões dos gases poluentes. Uma alternativa encontrada pelas montadoras é a construção de veículos que apresentam menor consumo de combustíveis, assim reduzindo as emissões de gases. De acordo com um estudo publicado pela consultoria McKinsey, Ealey e Mercer em 2002, no período de 1982 a 2002 as montadoras melhoraram significativamente a potência, consumo de combustível e emissão de poluentes de seus motores. A previsão é de que pelo menos até 2025, a utilização do motor a combustão interna seja ainda amplamente predominante para fins de transporte terrestre. Sendo esta afirmação também mencionada pelo diretor global de sustentabilidade e meio ambiente da Ford, John Viera, que prevê em 2020, apenas $25 \%$ dos carros vendidos pela companhia sejam híbridos ou elétricos [3].

Segundo Junior [4] o motor a combustão interna ainda tem ampla vantagem, pois conta com uma rede de abastecimento consolidada enquanto outras formas de energia teriam que construir essa rede. Embora a maioria da população apoie as restrições à emissão de poluentes, devido aos altos custos atuais de veículos menos poluentes, como os elétricos e híbridos, poucos consumidores podem e estão dispostos a pagar mais por isso, sabendo que irá sacrificar um pouco em potência e/ou conforto.

A fim de se obter resultados, contornando os problemas encontrados, novas tecnologias estão sendo pesquisadas e implementadas pelas montadoras, dentre elas, o downsizing (diminuição) do motor com utilização do Sistema de Indução 
Forçada (FIS), que está ganhando popularidade como uma solução viável e consiste em construir motores menores, que utilizam menos combustível, ou seja, motores menos poluentes, com turbo compressores, e com a potência semelhante de motores maiores.

Este estudo tem como objetivo obter um panorama atualizado sobre a utilização de turbo compressores para a construção de veículos automotores mais econômicos, não permitindo que haja uma perda significativa de potência.

Sendo os veículos automotores responsáveis por uma parte significativa da emissão de gases poluentes, a busca por tecnologias alternativas que possam diminuir as emissões tem se tornado mais frequentes. Com as novas regras e metas de emissões de gases poluentes a indústria automotiva fica à mercê da renovação tecnológica, desta forma buscar alternativas que atendam tanto as regras de combate à poluição e a expectativa do consumidor torna-se um desafio.

O sistema de indução forçada hoje é uma alternativa viável para atender tanto as metas de preservação ambiental, quanto a expectativa do consumidor em relação a potência do automóvel.

\section{INFORMAÇÕES GERAIS}

\subsection{Motores de Ciclo Otto}

A evolução tecnológica dos motores do ciclo Otto se dá pelo desenvolvimento dos fatores de produto e processo. O ciclo Otto foi desenvolvido por Nikolaus August Otto, documentado em 1867 e redefinido em 1876, ganhando inclusive medalha de ouro na Exposição de Paris de 1867, a versão final comercial era mais refinada e foi lançada em 1883 quando era chamado de 'motor de gás atmosférico' [1]. O motor de ciclo Otto se sobressaiu sobre seus demais concorrentes, motores elétricos e a vapor, principalmente com o apoio de uma ampla disponibilidade de postos de abastecimento instalados nos centros urbanos, principalmente a partir da década de 1920, quando o abastecimento deixou de ser realizado nas residências [5].

Este motor é considerado uma máquina térmica, sendo um dispositivo capaz de realizar trabalho, convertendo energia térmica em energia mecânica por meio da explosão da mistura de oxigênio com combustível no interior da sua 
câmara de combustão. É chamado de motor de quatro tempos, devido as suas quatro etapas de funcionamento, sendo elas admissão, compressão, explosão e exaustão.

No primeiro tempo, com o pistão em movimento descendente, dá-se a admissão, que se verifica, na maioria dos casos, por aspiração automática da mistura ar-combustível. No segundo tempo, ocorre a compressão, com o pistão em movimento ascendente. Pouco antes do pistão completar o curso, ocorre a ignição por meio de dispositivo adequado. No Terceiro tempo, com o pistão em movimento descendente, temos a ignição, com a expansão dos gases e transferência de energia ao pistão (tempo motor). E por fim, no quarto tempo, o pistão em movimento ascendente, empurra os gases de escape para a atmosfera [6].

Durante os quatro tempos, transmitiu-se trabalho ao pistão só uma vez. Para fazer com que as válvulas de admissão e escapamento funcionem corretamente, abrindo e fechando as passagens nos momentos exatos, a árvore de comando de válvulas (ou eixo de cames) gira a meia rotação do motor, completando uma volta a cada ciclo de quatro tempos. Com a realização de todas estas etapas, o torque é direcionado para as rodas por meio de eixos, transmissões e caixas diferenciais, permitindo assim, o movimento do veículo [6].

\subsection{Emissão de Poluentes e Suas Causas}

O trânsito de veículos é um grande colaborador nas emissões de contaminantes, principalmente nos grandes centros urbanos, onde o acúmulo de carros é mais frequente, ou em áreas onde ainda são usados combustíveis com elevado conteúdo de enxofre. A utilização em larga escala dos motores de combustão interna possibilita a liberação de gases na atmosfera, tais como óxido de nitrogênio, monóxido de carbono, dióxido de carbono, chumbo, entre outros, sendo estes extremamente prejudiciais ao meio ambiente e à saúde da população em geral. É liberado também dióxido de nitrogênio (NO2), que por sua vez contribui para a formação de oxidantes fotoquímicos como o ozônio [7]. 
Segundo Loureiro [8], as emissões de origem veicular são resultado da combustão do combustível ou de sua evaporação e de acordo com o Plano de Controle da Poluição por Veículos em Uso - PCPV (2005) os principais poluentes lançados na atmosfera pelos veículos automotores são provenientes do processo de combustão incompleta, estes causados por algum fator prejudicial ao perfeito funcionamento do motor e a realização de seu processo de combustão de maneira completa.

Cada um desses poluentes é emitido em maior ou menor quantidade em função do combustível utilizado, do tipo de motor, da sua regulagem, da manutenção e modo de dirigir. Em veículos novos essas emissões foram bastante controladas com a adição de certas tecnologias (catalisadores, injeção eletrônica de combustível entre outros). Apesar de, individualmente, esse tipo de emissão ser aparentemente insignificante, ao se analisar o número de veículos existentes nas grandes cidades, verifica-se a geração de toneladas de poluentes por dia [8].

\subsection{Turbo Compressor}

O turbo compressor utilizado pela indústria automotiva foi construído e patenteado por Alfred Büchi em 1905, descrita como uma máquina pela qual a energia dos gases expelidos pelo motor aumentaria o fluxo da mistura arcombustível no qual a energia cinética dos gases de escape moveria um eixo ligado a uma turbina, que serviria como compressor para o ar admitido pelos cilindros [9].

A turbina utiliza a energia cinética e a energia térmica oriunda dos gases de escape do motor para acionar o compressor, que através de um eixo transfere esta energia ao compressor localizado na admissão do motor. Ao atingir determinada rotação e carga, o compressor começa a gerar pressão positiva no coletor de admissão, aumentando a massa de ar que o motor admite por ciclo, acrescentando potência ao motor, devido ao aumento de entropia.

\subsubsection{Sistema de Indução Forçada (FIS)}

O sistema de indução forçada (FIS) é constituído basicamente por uma turbina, um turbocompressor e um intercooler. Seu objetivo nada mais 
é do que injetar sob uma determinada pressão, uma maior quantidade de $\operatorname{ar}\left(\mathrm{O}_{2}\right)$ que será utilizado na combustão interna no motor.

Dijkstra et al. [10] descreve que em seu modelo de construção mais simples, os gases que seriam eliminados pelo escapamento do veículo são canalizados para uma turbina, fazendo a mesma girar. A turbina por sua vez fará com que o turbo compressor se movimente por meio de um eixo entre eles. $O$ turbo quando em movimento, succiona $o$ ar do ambiente e o direciona sob alta pressão até o intercooler, onde passará por um processo de resfriamento e logo depois seguirá até a câmara de combustão. $\mathrm{O}$ aumento de $\mathrm{O}_{2}$ na câmara de combustão permite uma maior taxa de explosão interna, o que consequentemente resulta no aumento da potência.

Este mecanismo permite fazer uso dos gases que seriam descartados sem qualquer utilidade para ajudar a prover maior eficiência e potência para o motor, podendo assim, construir um motor de menor cilindrada e com menos consumo de combustível, porém possuindo um desempenho semelhante aos demais motores com maiores tamanhos e com maiores taxa de consumo de combustível.
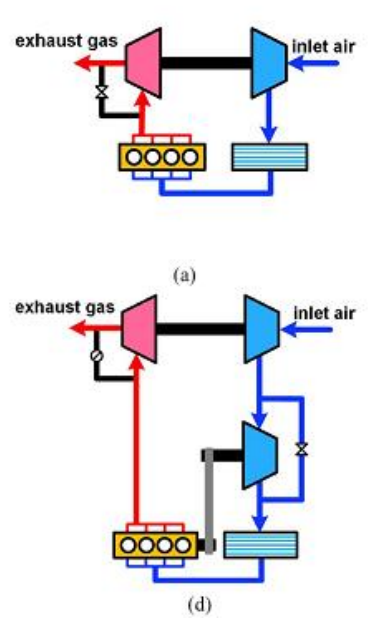
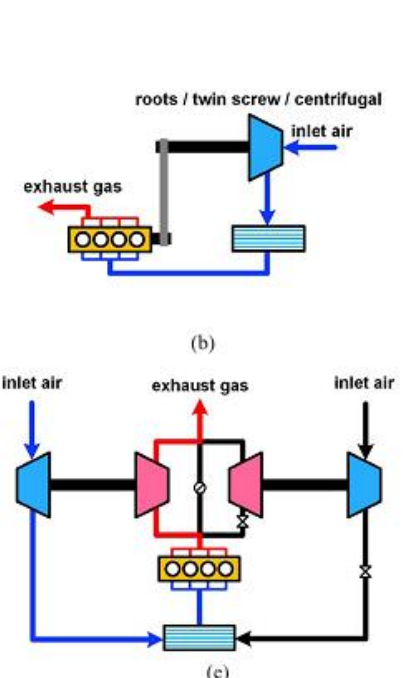
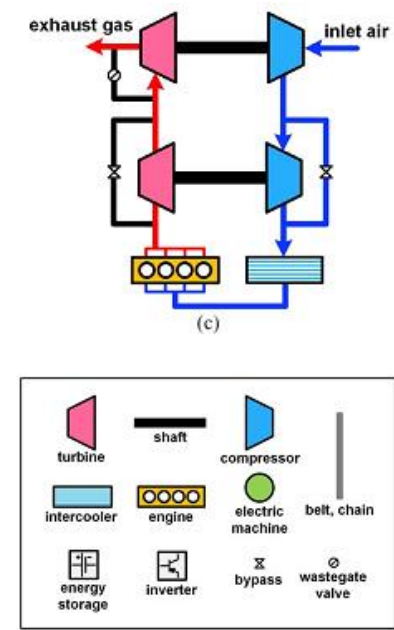

Figura 1 - Possíveis métodos de instalação do sistema FIS

Nos modos de instalação mostrados anteriormente, há um problema denominado "Turbo Lag", que consiste em um atraso de resposta do turbo devido à uma baixa quantidade de gases expelidos pelo motor, 
devido a uma baixa rotação por exemplo, podendo ocasionar uma resposta não tão imediata quanto se deseja naquele instante.

\subsubsection{Sistema de Indução Forçada Elétrica (EFIS)}

Com o objetivo de diminuir o efeito do chamado "Turbo Lag", uma máquina elétrica de alta velocidade é interligada entre a turbina e o compressor. Quando a velocidade do motor é baixa, a máquina elétrica funciona como um motor que fornece torque adicional ao compressor, gerando pressão de reforço mais alta com uma resposta transitória mais rápida. Dessa maneira, permitindo uma resposta mais rápida e eficaz do sistema até mesmo em baixas rotações do motor.

Assim como no FIS, o EFIS também possui diversos modos de instalação, dependendo da necessidade em específico. Segue uma figura ilustrativa dos possíveis métodos de instalação.
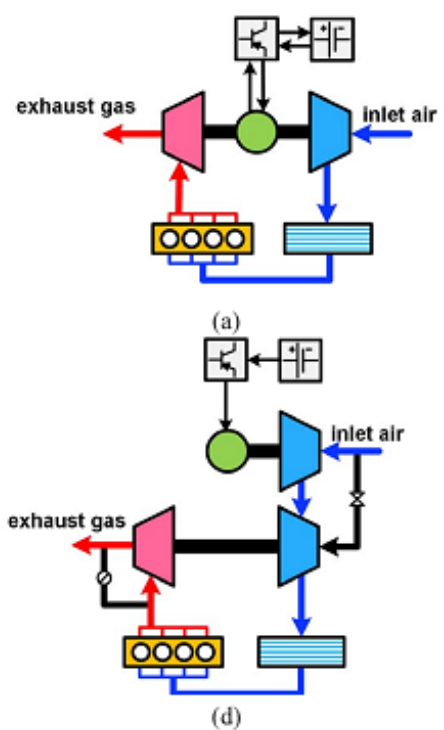

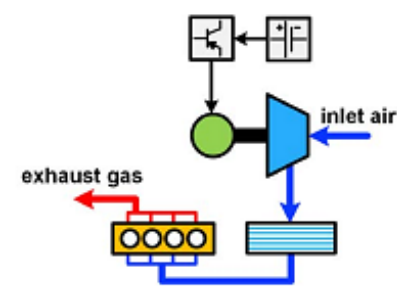

(b)

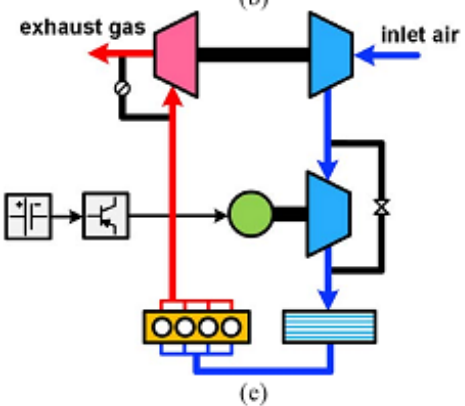

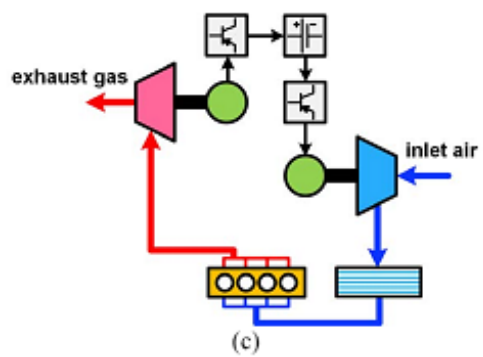

(c)

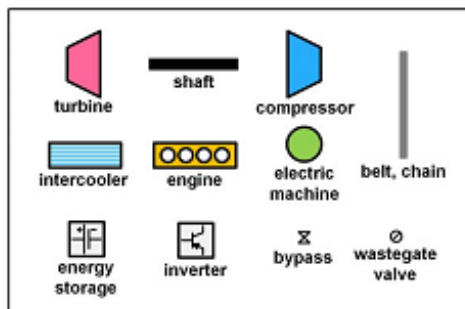

Figura 2 - Possíveis métodos de instalação do sistema EFIS

Para se comprovar que a velocidade de resposta transitória do sistema EFIS é maior do que a do FIS, é mostrado matematicamente que a relação torque/inércia do EFIS se demonstra maior que a do FIS convencional: 


\section{$\frac{T_{\text {EFIS_net }}}{J_{\text {EFIS }}}>\frac{T_{\text {NFIS_net }}}{J_{\text {NFIS }}}$}

Equação 1 - Relação torque/inércia

Onde:

TEFIS_net $=$ Torque do EFIS

TNFIS_net= Torque do FIS

JEFIS = Inércia do EFIS

$J_{\text {NFIS }}=$ Inércia do FIS

Normalmente, a resposta transitória pode ser significativamente melhorada com o EFIS, mas depende principalmente da quantidade de energia elétrica disponível no sistema elétrico do veículo e na máquina elétrica. A aplicação de tal sistema se faz muito interessante em motores de pequeno porte, que normalmente teriam uma baixa potência quando em baixas rotações, não sendo atrativos para o exigente mercado atual.

\subsection{Possíveis Problemas}

Para Lee et al. [11], a utilização de um sistema de indução forçada de maneira incorreta no motor, sem os cuidados necessários de compatibilidade entre eles, pode acarretar uma série de problemas, sejam eles mecânicos ou até mesmo elétricos. Toda máquina elétrica possui sua faixa de frequência na qual irá trabalhar de maneira adequada, a frequência fundamental de uma máquina elétrica usada em um turbo compressor elétrico varia de 1 a $8 \mathrm{kHz}$, dependendo da velocidade nominal e do número de pólos. Porém, a frequência de comutação de um acionamento motorizado pode exceder 20 $\mathrm{kHz}$ [12]. Esta grande diferença resulta em diversos problemas em dispositivos de comutação de energia e acionamentos de motor, como alta perda de comutação, dissipação de calor e interferência eletromagnética. Sendo assim, um grande desafio para as empresas automobilísticas realizar 
tal sincronização de frequência entre a máquina elétrica do EFIS e o sistema elétrico do veículo.

A implantação de um sistema FIS em um motor de combustão interna também exige atenção quanto à construção mecânica das partes tanto do sistema, quanto do motor. Tais cuidados devem ser tomados para evitar problemas como fadiga mecânica do motor, danos aos rolamentos, danos devido a temperatura, entre outros [13].

A fadiga mecânica do motor pode ocorrer devido a uma maior exigência e maiores tensões internas nas peças, gerando um desgaste prematuro dos componentes mecânicos que não foram devidamente projetados para resistir às novas taxas de pressões e altas rotações que o sistema estará sujeito. Rolamentos também estão sujeitos a diminuição da vida útil devido às altas rotações em que serão submetidos, sendo necessário que sejam cada vez mais resistentes e com melhores lubrificações. Como alternativa de solução, empresas atualmente já fazem uso de rolamentos magnéticos, pois estes não apresentam desgaste e perdas devido ao atrito em seu interior [14].

Por fim, a temperatura interna do motor também tem sido um fator importante na hora de projetar peças que irão ser utilizados em sistemas FIS, pois devido ao aumento de pressão, também ocorrerá o aumento de temperatura, se fazendo necessário o uso de bons equipamentos de refrigeração e materiais cada vez mais resistentes à altas temperaturas [15].

\section{CONCLUSÃO}

Com a análise dos estudos do funcionamento e da implementação dos turbos compressores, temos uma solução parcial viável para os problemas de emissão de poluentes na atmosfera, pois permitem construir motores menores com potências similares a motores maiores. Motores menores consomem menos combustível, logo, são menos poluentes, além de seu procedimento de fabricação ser mais econômico.

No presente, os motores híbridos e elétricos, apesar de serem mais ecologicamente corretos quando comparados com motores turbos, ainda são alternativas mais caras para o consumidor final que visa diminuir a poluição ambiental, portanto, tais soluções ainda são pouco utilizadas no mercado brasileiro, o que resulta na alta popularidade atual dos motores turbo. 


\section{REFERÊNCIAS}

[1] TAMINIAU, Y. Beyond known uncertainties: interventions at the fuel-engine interface. Research Policy, v. 35, 2006.

[2] DENATRAN, Frota 2011. Disponível em: <www.denatran.gov.br/frota.htm> Acesso em: 09 Julho de 2018.

[3] MAUTONE, S. Entrevista: John Viera. O Estado de S. Paulo. São Paulo, 17 dez. 2011.

[4] JUNIOR, W. G. A evolução da tecnologia de motores: um estudo preliminar. XV SEMEAD. São Paulo. 2012.

[5] MELAINA, M. W. Turn of the century refueling: a review of innovations in early gasoline refueling methods and analogies for hydrogen. Energy Policy, $v$. 35, 2007.

[6] STONE, R. Internal Combustion Engines. Society of Automotive Engineers, Inc. 2 ed Warrendale, PA, USA. 1993.

[7] TUNDO, P.; ZECCHINI, F. Mudanças climáticas globais. Consórcio InterUniversitário Nacional"A Química para o Ambiente" e IUPAC União Internacional de Química Pura, Julho de 2007.

[8] LOUREIRO L. N.; Panorâmica Sobre Emissões Atmosféricas Estudo De Caso: Avaliação Do Inventário Emissões Atmosféricas da Região Metropolitana Do Rio De Janeiro Para Fontes Móveis. Tese Universidade Federal do Rio de Janeiro, 2005.

[9] BÜCHI, A. Verbrennungskraft maschinenanlage. 204630. Kaiser liches Patentamt. Alemanha, v. 16, 1905.

[10] DIJKSTRA, R., BOOT, M., EICHHORN, R., SMEULDERS, D., LENNBLAD, J., SERRARENS, A. Experimental analysis of engine exhaust waste energy recovery using power turbine technology for light duty application. Society of Automotive Engineers. Int. J. Engines, 2012.

[11] LEE W., SCHUBERT E., LI, Y. LI, S., BOBBA, D., SARLIOGLU, B. Overview of Electric Turbocharger and Supercharger for Downsized Internal Combustion Engines. IEEE Transactions OnTransportation Electrification. Março 2017.

[12] KIM, S., SEOK, J. K. Comprehensive PM motor controller design for electrically assisted turbo-chargersystems. IEEE Energy Convers Expo. Setembro, 2013. 
[13] SEBASTIAN, T. Temperature effects on torque production and efficiency of PM motors using NdFeB magnets. IEEE Trans. Ind. Appl. Março/Abril, 1995.

[14] SULAIMAN E., KOSAKA, T., MATSUI, N. Parameter optimization study and performance analysis of 6S-8P permanent magnet flux switching machine with field excitation for high speed hybrid electric vehicles. Eur. Conf. Power Electron. Appl., p. 1-9, Agosto/Setembro, 2011.

[15] LI, S., LI, Y., CHOI, W., SARLIOGLU, B. High-speed electric machines: Challenges and design considerations. IEEE Trans. Transport. Electrific. Março, 2016. 